\title{
2. SOLAR ABUNDANCES OF THE H, C, N AND O ATOMS \\ DEDUCED FROM MOLECULAR ABUNDANCES
}

\section{By J. Hunaerts \\ Observatoire Royal de Belgique}

$A$ priori it would seem possible to determine the solar abundances of the $\mathrm{H}, \mathrm{C}, \mathrm{N}$ and $\mathrm{O}$ atoms on the basis of the solar abundances of the $\mathrm{CN}, \mathrm{CH}, \mathrm{C}_{2}, \mathrm{NH}$ and $\mathrm{OH}$ molecules. Such a determination entails two distinct problems:

(I) The determination of the molecular abundances with the help of the measured intensities of individual rotational lines.

(2) The calculation of atomic abundances on the basis of the equations of dissociation equilibrium.

(I) Molecular abundances. We have recently(x) estimated the solar abundances of $\mathrm{CN}, \mathrm{CH}, \mathrm{C}_{2}, \mathrm{NH}$ and $\mathrm{OH}$. For this purpose we have measured the equivalent widths of a large number of rotational lines of $\mathrm{CN}, \mathrm{CH}$ and $\mathrm{C}_{2}$, using Minnaert's Photometric Atlas of the Solar Spectrum. The Nf-values obtained in this way enabled us to draw the curves of growth for these molecules. In the case of $\mathrm{NH}$ and $\mathrm{OH}$ the Rowland intensities were converted into equivalent widths by using a calibration by Roach and Phillips (2) and the Nf-values were deduced from the curves of growth thus established.

We calculated in the usual manner the molecular excitation temperatures which turned out to be of the order of $4500^{\circ} \mathrm{K}$., hence correspond to the temperature of the solar photosphere at very small optical depth.

The conversion of the Nf-values into the absolute molecular abundances, requires the knowledge of the oscillator strengths $f$, on which a great deal of doubt still exists. There are experimental determinations of $f(\mathrm{CN})$ and $f(\mathrm{OH})$ which enabled Lyddane, Rogers and Roach (3) to compute the $f$-values for $\mathrm{C}_{2}, \mathrm{CH}$ and NH. Moreover, Mrs Rieke (4) has estimated theoretically the $f$-values for $\mathrm{OH}, \mathrm{CH}$ and $\mathrm{NH}$.

$\begin{array}{lcc} & \text { Lyddane } \text { et al. } & \text { Mrs Rieke } \\ \mathrm{CN}\left({ }^{2} \Sigma^{+}-{ }^{2} \Sigma^{+}\right) & 2 \cdot 6 \times 10^{-2} & - \\ \mathrm{C}_{2}\left({ }^{3} \Pi_{g}-{ }^{3} \Pi_{24}\right) & 2 \cdot 4 \times 10^{-2} & - \\ \mathrm{OH}\left({ }^{2} \Sigma-{ }^{2} \Pi_{i}\right) & 3.0 \times 10^{-4} & 4 \times 10^{-4} \\ \mathrm{CH}\left({ }^{2} \Delta-{ }^{2} \Pi\right) & 4 \cdot 5 \times 10^{-4} & 10^{-2} \\ \mathrm{NH}\left({ }^{3} \Pi_{i}-{ }^{3} \Sigma^{-}\right) & 7.2 \times 10^{-4} & f(\mathrm{CH})>f(\mathrm{NH})>f(\mathrm{OH})\end{array}$

The abundances computed on the basis of the $f$-values of Lyddane, Rogers and Roach are

$\begin{array}{cccccc} & \mathrm{CN} & \mathrm{CH} & \mathrm{C}_{2} & \mathrm{NH} & \mathrm{OH} \\ \log \mathrm{S} & 16.32 & 17.31 & 14.96 & 16.98 & 17.52\end{array}$

At this point it should be made clear that the adopted $f$-values may be wrong by appreciable factors.

(2) Atomic abundances. The relative atomic abundances of $\mathrm{H}, \mathrm{C}, \mathrm{N}$ and $\mathrm{O}$ may in principle be deduced from the molecular abundances on the basis of the classical equations of dissociation equilibrium

$$
p(A) p(B)=K p(A B)
$$

the $p$ 's being the partial pressures and $K$ the dissociation constant. This has been done, following Russell's classical schematic procedure. Serious uncertainties already arise from the astronomical nature of the problem, since an idealized atmosphere has to be adopted. But additional uncertainties arise from inadequate data on several dissociation energies which play a role in the dissociation equilibrium. We have adopted the dissociation energies recently published by Gaydon(s).

$$
\begin{array}{llllll}
D \text { in e.V. } & \mathrm{CN}=7 \cdot 6 & \mathrm{C}_{2}=5 & \mathrm{CH}=3.47 & \mathrm{NH}=3 \cdot 6 \mathrm{I} & \mathrm{OH}=4.34 \\
& \mathrm{CO}=\mathrm{II} \cdot \mathrm{II} & \mathrm{N}_{2}=9.764 & \mathrm{NO}=6.49 & \mathrm{H}_{2}=4.4776 & \mathrm{O}_{2}=5.084
\end{array}
$$


On this basis the atomic abundances turn out to be:

$$
\begin{array}{lll}
\log T(\mathrm{H})=23 \cdot 97 & & T(\mathrm{H})=\text { ro,000 } \\
\log T(\mathrm{C})=21 \cdot 03 & T(\mathrm{C})= & \text { II.5 } \\
\log T(\mathrm{~N})=20 \cdot 99 & T(\mathrm{~N})= & \text { I0.5 } \\
\log T(\mathrm{O})=21 \cdot 19 & T(\mathrm{O})= & \text { I7 }
\end{array}
$$

Since these values depend in a complicated fashion on the $f$-and $D$-values, it is impossible to estimate the accuracy of the calculated figures. If the values of the dissociation energies given by Herzberg (6) $\left[D(\mathrm{CN})=5.95\right.$ e.V.; $\left.D\left(\mathrm{C}_{2}\right)=3.6 \mathrm{e} . \mathrm{V}.\right]$ were adopted, much higher values of the abundances of $\mathrm{C}, \mathrm{N}$ and $\mathrm{O}$ relative to $\mathrm{H}$ would be obtained: this is due to the essential role of $D(\mathrm{CN})$ in the calculations.

Slight changes in the $f$-values would give the abundance ratio of $\mathrm{C}$ and $\mathrm{N}$ obtained by Bethe.

\title{
CONCLUSIONS
}

I. The determination of molecular excitation temperatures gives consistent results. We have to admit low temperatures for the photospheric region of effective absorption of the faint molecular lines.

2. The estimated atomic abundances are very uncertain, owing mainly to the uncertainties of the $f$-and $D$-values. Experimental and theoretical work in this field is highly desirable.

\section{BIBLIOGRAPHY}

(I) J. Hunaerts. Ann. d'Astrophysique, ro, 237, 1947.

(2) F. E. ROACH and J. G. Phillips. Ap. J. 96, 71, 1942.

(3) R. H. Lyddane, F. T. Rogers and F. E. Roach, Phys. Rev. 6o, 28I, I94 I.

(4) C. A. Rieke. Pop. Astr. 49, 523, I94 I.

(5) A. G. GaYdon. Dissociation Energies. London, I947.

(6) G. Herzberg. Molecular Spectra and Molecular Structure. I. Diatomic Molecules. New York, I939.

\section{THE CHEMICAL COMPOSITION OF STELLAR ATMOSPHERES}

\author{
By A. UnsöLD \\ Astronomical Observatory, Kiel, Germany
}

The quantitative analysis of stellar atmospheres requires-if we may put it somewhat paradoxically-already considerable knowledge of their composition and structure. In practice it is therefore necessary to begin with the simplest problems and even there to proceed in successive approximations.

Let us first consider normal atmospheres which are completely determined by stating their chemical composition, effective temperature $T_{e}$ and surface gravitation $g$. We divide them best into three groups:

I. Early spectral types from $\mathrm{O}$ to $\mathrm{A}_{5}$ : completely ionized. Continuous absorption by hydrogen and helium atoms. Scattering by free electrons.

II. Medium spectral types from $\mathrm{A}_{5}$ to $\mathrm{K}_{5}$ : hydrogen neutral. $\mathrm{H}^{-}$continuum.

III. Late types from $\mathrm{K}_{5}$ on: formation of molecules; molecular spectra.

We shall deal here mainly with early spectral types, the Sun as chief representative of group II being treated by Prof. Minnaert. Then I shall say something about peculiar spectra and finally add a few considerations on the methods which seem to be important for a successful development of our research. 